\title{
Atrio-Ventricular Junction Behaviour During Atrial Fibrillation
}

\author{
P Bonizzi, V Zarzoso, O Meste \\ I3S Laboratory, University of Nice - Sophia Antipolis, France
}

\begin{abstract}
Up to now the functioning of the Atrio-Ventricular Junction (AVJ) during Atrial Fibrillation (AF) is still not completely understood. To shed some light on the AVJ behavior during AF episodes, this study analyses the existence of a possible relationship between the occurrence of a heart beat and the power of the atrial activity $(A A)$ preceding this heart beat in an electrocardiogram (ECG) signal. $A A$ power is measured in the interval of the ECG between the onset of the heart beat under analysis and the ending of the previous heart beat (T-Q interval). Our analysis has shown a difference in the distributions of AA power versus cardiac cycle length (RR interval on the ECG) between healthy and pathological subjects. In particular, a negative trend between these two parameters is discovered in pathological subjects. This negative relation shows a possible coherence between power arriving at the AVJ and the triggering of the heart beat, which is in line with the assumptions made in the quantitative model for the ventricular response during AF by Cohen [1].
\end{abstract}

\section{Introduction}

Atrial Fibrillation (AF) represents the most common sustained cardiac arrhythmia in adults. It consists of a misfunction of the atrium characterized by a modification of the normal atrial activity (AA) pattern on the electrocardiogram (ECG) signal. Epidemiologic studies have shown that its prevalence and incidence doubles with each advancing decade beyond 50 years reaching $10 \%$ in people over 80 and has direct impact on mortality and morbidity $[2,3]$.

Although the mechanism and effective treatments for most other supraventricular tachyarrhythmia have been discovered, the understanding of AF remains incomplete. In particular, no unifying mechanism has been found yet to explain the behaviour of the atrio-ventricular junction (AVJ) during AF. It is well known that heart rate variability is enhanced during AF [1, 4], while other studies have revealed a more complex relationship between AVJ behaviour and AF [5]. In addition, the variation of the RR interval during AF has been thought to result mainly from autonomic modulation of the electrophysiological properties of the atria and the AVJ [6]. Therefore, the characterization of the ventricular rhythm in AF has been controversial and its mechanism has been a subject of debate for decades [7], thus revealing that the prevailing ventricular rate during high atrial rate is a complex dynamic parameter.

Different hypothesis were introduced to explain the irregular ventricular response during AF, i.e., the dependence of the decremental conduction and repetitive concealment on the AF impulses within the AVJ [8], or yet the dependence of the electrotonic modulation on the AVJ propagation by concealed AF impulses [9].

Several quantitative models for the ventricular response during AF were developed $[1,7,10]$ to deal with this problem. The AVJ model presented by Cohen et al. [1] introduced the hyphotesis that it can be treated as a lumped structure with well defined electrical properties, including the refractory period, the automaticity and a defined depolarization threshold. From this point, the present work analyzes the presence of a possible correlation between the occurrence of a heart beat and the power of the AA observable in the ECG signal during the T-Q interval preceding this heart beat. This analysis aims to test the presence of a dependence between the AA power arriving at the AVJ and the RR period, following the idea that the AVJ generates an activation wave when the AA power arriving at it exceeds a threshold value, intrinsic to the AVJ.

The study of AF and its characteristics can to a large extent be carried out through the analysis of the surface electrocardiogram (ECG), which has the advantage to be a noninvasive technique and is already reported to be useful [11].

\section{Methods}

A dataset composed of 23 real recordings ( 2 healthy subjects and 21 presenting AF) was employed to analyze the proposed idea. All signals were recorded and digitized at a sampling rate of $1 \mathrm{KHz}$. Among the segments em- 


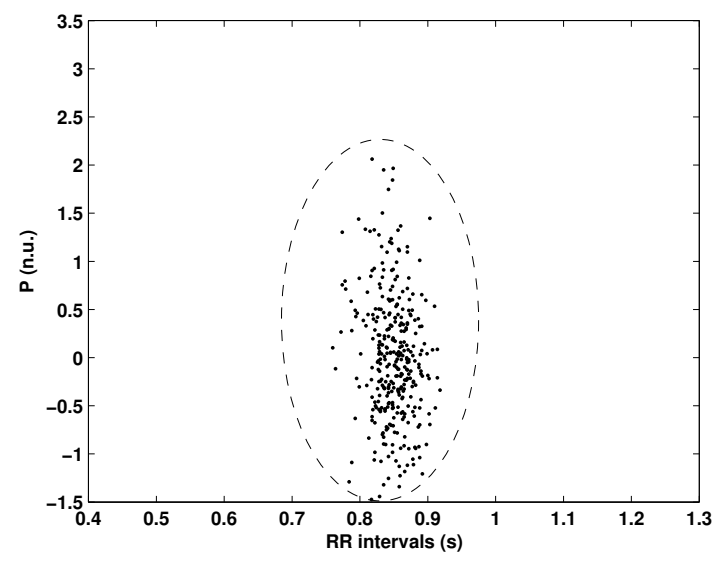

Figure 1. AA power versus RR interval distribution for a healthy subject. The shape distribution does not show the presence of any particular relation; n.u., normalized units; $\mathrm{s}$, seconds.

ployed in this analysis 19 were recorded using a standard 12-lead derivation system of 12s length (all AF) while 4 were recorded using a 9-lead derivation system of 300s length ( 2 healthy and 2 displaying AF). Pre-processing was done by applying a zero-phase low pass filter to remove physiologically irrilevant low frequency signal variations $(<1 \mathrm{~Hz})$ [12].

To allow the calculation of the AA power in each T-Q segment the algorithm of Zong et al. was employed to detect $Q$ wave onset and $T$ wave ending in each heart beat [13]. AA power in the T-Q interval referred to the $i$ th heart beat on the $l$ th lead was calculated as follows:

$$
P_{A A_{i}}^{(l)}=\frac{1}{\#\left[Q_{i}-T_{i-1}\right]} \sum_{n=T_{i-1}}^{Q_{i}} x_{l, n}^{2}
$$

where $x_{l}$ is the digitized ECG signal recorded by the $l$ th lead, $T_{i-1}$ is the $\mathrm{T}$ wave ending instant of the $(i-1)$ th heart beat, $Q_{i}$ is the $\mathrm{Q}$ wave onset instant of the $i$ th heart beat (the beat under analysis) and $Q_{i}-T_{i-1}$ is the number of samples contained in the $i$ th T-Q interval.

Once each T-Q interval has been selected, AA power is calculated for each of all the leads $\left(P_{A A_{i}}^{(l)}\right.$, with $l$ generic lead, $i$ generic beat).

To make use of the AA power of all leads, a normalization step is required. This is because a lead placed on the body surface records an electric activity whose amplitude depends on the distance between the electrode and the location of the recorded activities, on the electrical properties of the tissues and on the directions of the activities. Therefore, in each specific lead AA powers for all the T-Q segments are standardized in order to get a zero-mean and

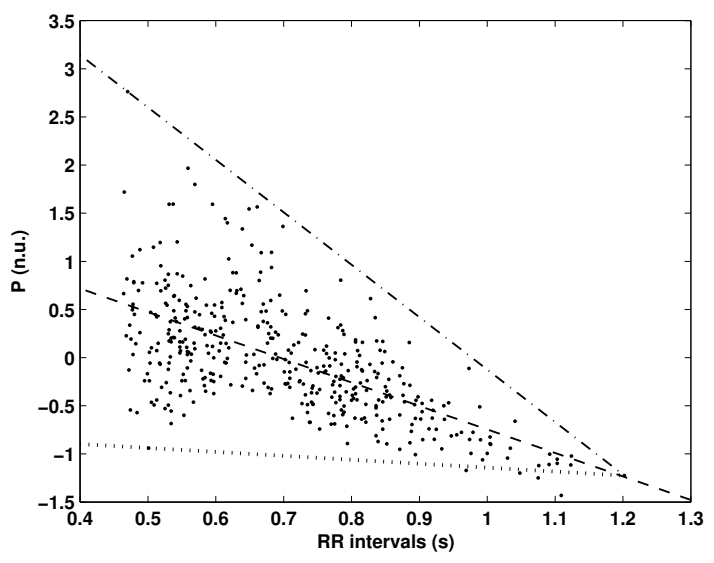

Figure 2. AA power versus RR interval distribution for a pathological subject. The shape distribution shows the presence of an inverse relation (dashed line), even if it is quite complex, due to the dispersion of the distribution (locked up between dotted and dashed-dotted lines); n.u., normalized units; s, seconds.

unit-variance normalization of power measurements on a lead-by-lead basis. This means that each AA power value is subtracted by the AA power mean value in that lead and devided by its standard deviation, as follows:

$$
\tilde{P}_{A A_{i}}^{(l)}=\frac{P_{A A_{i}}^{(l)}-\overline{P_{A A}^{(l)}}}{\sigma_{P_{A A}^{(l)}}}
$$

where $\tilde{P}_{A A_{i}}^{(l)}$ is the normalized AA power of the T-Qith interval, $\bar{P}_{A A}^{(l)}$ is the AA power mean value of the segments in the lead under analysis and $\sigma_{P_{A A}^{(l)}}$ is its standard deviation.

Subsequently, the mean value of the normalized AA powers occurring at the same heart beat in all leads is calculated for each heart beat as follows:

$$
\overline{\tilde{P}_{A A_{i}}}=\frac{1}{L} \sum_{l=1}^{L} \tilde{P}_{A A_{i}}^{(l)}
$$

where $L$ is the total number of leads. It is easy to see that $\tilde{P}_{A A_{i}}$ is a summary of the whole AA preceding the $i$ th heart beat. This normalizing procedure is necessary for exploiting at one time the spatial information contained in each lead as correctly as possible, keeping into account the arbitrary spatial differences among each electrode, as previously mentioned.

\section{Results}

The proposed approach for the analysis of AA power in the T-Q interval versus RR period was applied using a pa- 


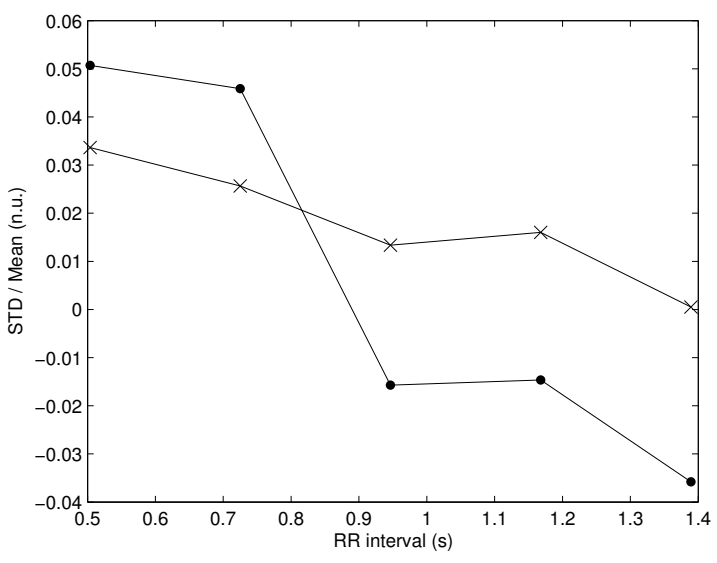

Figure 3. AA power meanvalues (point-marked line) and standard deviations ( $\mathrm{x}$-marked line) for all the pathological subjects split in five RR bins. Each point fixed in correspondence to the RR meanvalue of each bin; n.u., normalized units; s, seconds.

tient dataset of 23 recordings. Fig. 1 and 2 plot the mean values of the normalized AA powers $\overline{\tilde{P}_{A A_{i}}}$ versus the $R R_{i}$ intervals for a healthy and an unhealthy subject, respectively.

First of all, the difference in the $\overline{\tilde{P}_{A A_{i}}}=f\left(R R_{i}\right)$ shape distribution between healthy and pathologic subjects is remarkable. The healthy subject shows a rounded distribution, as in Fig. 1, while the pathological subjects seem to exhibit a triangular shape distribution, summarized by the dotted and dashed-dotted lines in Fig. 2. For pathological subjects an inverse relation appears between AA power arriving at the AVJ before each heart beat and the corresponding RR period (dashed line in Fig. 2). However, from the spread of the distribution it can be derived that it is not a linear relation. Possible explenations of this tendency are suggested in the discussion part of this paper.

Fig. 3 summarizes the general behaviour of the $\overline{\tilde{P}_{A A_{i}}}=$ $f\left(R R_{i}\right)$ relation for all the pathological subjects under analysis. Indeed, it shows the trend of AA power mean values and standard deviations respectively, versus the RR intervals, after grouping RR intervals in five different classes and keeping the mean value of the $\overline{\tilde{P}_{A A_{i}}}$ (point-marked line) and of its standard deviations (x-marked line) in each class for all the subjects. Both the curves show clearly the inverse relation between AA power and RR interval.

\section{Discussion and conclusions}

In this study, the existence of a relationship between the occurrence of a heart beat and the power of the AA pre- ceding this heart beat has been analyzed. First of all, the importance of a normalization step has been described, to give the possibility to exploit the spatial information kept in all leads, since the amplitude of the signal recorded by one lead can not be compared directly to that of another one.

The analysis of the $\overline{\tilde{P}_{A A_{i}}}=f\left(R R_{i}\right)$ relationship has shown a difference between healthy subjects, for which the presence of a relation is not evident (uniform scatter plot in Fig. 1), and pathological subjects, for which an inverse relation between these two parameters seems to be present (dashed line in Fig. 2), even if the distribution is quite spread (triangular scatter plot in Fig. 2). The presence of this relation is supported by the fact that the probability of having high AA power values associated to short RR intervals is higher than for long RR intervals, as clearly shown in Fig. 3. This finding suggests that the AVJ behaviour during $\mathrm{AF}$ is characterized by a power threshold value (in analogy with the electric depolarization threshold value), for which the likelihood of generating a cardiac beat by the AVJ is related to the amount of AA power carried by the AF activity arriving at it, in agreement with the AVJ models presented by Cohen et al. [1] and by Lian et al. [7]. Therefore, the higher the AA power arriving at the AVJ in a defined time, the higher the possibility to go over the threshold and to generate a beat (related to a shorter RR interval). The results shown in the previous section (Fig. 3 ), in agreement with these theoretical models, suggest that $\mathrm{AF}$ impulses arrival to the $\mathrm{AVJ}$ is a dominant factor in the AVJ excitation.

However, the great variance of $\overline{\tilde{P}_{A A_{i}}}=f\left(R R_{i}\right)$ distribution for short RR periods in the pathological subjects seems not to support the hypothesis of an inverse relation (behaviour summarized by the dotted line in Fig. 2). This can be explained in different ways. First, if it could be supposed that the leads placed on the body surface are not able to capture completely the three dimensionsal field related to AA, together with the fact that AF works randomly in time and space, then it can be assumed that what is recorded is sometimes only a partial vision of what is really happening, due to the leads' spatial resolution limit. Therefore, if a low AA power value associated to a short RR interval is observed (instead of a longer one, as would be suggested by the inverse relation), it can be supposed that the electrical activity recorded at that moment on the ECG is only a reduced portion of the real one (leads' placement unable to capture the main direction of $\mathrm{AF}$ ). If this hyphothesis is correct, AA power in that particular moment must be actually greater, rising the related point in the $\overline{\tilde{P}_{A A_{i}}}=f\left(R R_{i}\right)$ plot nearer to the inverse relation (dashed line in Fig. 2). Thus, both the inverse relation and the hypothesis of dominance of $\mathrm{AF}$ impulses arriving at $\mathrm{AVJ}$ in generating its excitation are strengthened. On the other 
hand, we could think the opposite,i.e., that leads placement works well in capturing all the AF electrical activity in any direction. Consequently, power variance trend in the $\overline{\tilde{P}_{A A_{i}}}=f\left(R R_{i}\right)$ distribution could be due to the amount of noise present in the AA signal and to the way the power is computed (the longer the RR periods for which AA energy is divided the lesser the variance of the estimator of the AA power mean). On the contrary, if the amount of noise in the AA signal is unimportant, it means that other factors must be taken into account for the understanding of the spread of the variance and the AVJ way of functioning.

Also, it must be taken into account that there is no certainty about the fact that AA recorded in the ECG before a heart beat is effectively what reachs the AVJ. Indeed, it is the global activity of the heart in a particular moment. The portion of this global activity that really arrives at the AVJ depends on the AA waves interaction, and so on their level of spatial and temporal organization.

In conclusion, the frequency content of AA is known to provide important physiological and clinical informations about AF [11]. This study has evidenced that alternative information such as the amplitude (power) of the AA signal can also shed some light on the understanding of the physiological mechanism behind this condition. Future work aims to analyze more deeply this finding, trying to exploit also the AA masked by the QRS complex $[14,15]$.

\section{Acknowledgements}

The authors would like to express their gratitude to Leif Sörnmo and Francisco Castells for providing the real data. The work of Bonizzi Pietro is supported by the EU by a Marie-Curie Fellowship (EST-SIGNAL program : http://est-signal.i3s.unice.fr) under contract No MEST-CT2005-021175.

\section{References}

[1] Cohen RJ, Berger RD, Dushane TE. A quantitative model for the ventricular response during atrial fibrillation. IEEE 1983;781-796.

[2] Kannel WK, Abbott RD, Savage DD, McNamara PM. Epidemiologic features of chronic atrial fibrillation: the framingham study. N Engl J Med 1982;306:1018-22.

[3] Krahn AD, Manfreda J, Tate RB, Mathewson FA, Cuddy TE. The natural history of atrial fibrillation: incidence, risk factors, and prognosis in the Manitoba Follow-Up Study. Am J Med 1995;98:476-84.

[4] Fuster V, Ryden LE, Asinger RW. ACC/AHA/ESC guidelines for the management of patients with atrial fibrillation. JACC 2001;38:1266i-12661xx.

[5] Garrigue S, Tchou PJ, Mazgalev TN. Role of the differential bombardment of atrial inputs to the atrioventricular node as a factor influencing ventricular rate during high atrial rate. Cardiovasc Res 1999;44:344-355.
[6] Toivonen L, Kadish A, Kou W, Morady F. Determinants of the ventricular rate during atrial fibrillation. J Am Coll Cardiol 1990;16:1194-1200.

[7] Lian J, Mussig D, Lang V. Computer modeling of ventricular rhythm during atrial fibrillation and ventricular pacing. IEEE Trans Biomed Eng 2006;53:1512-20.

[8] Watanabe $Y$, Watanabe M. Impulse formation and conduction of excitation in the atrioventricular node. J Cardiovasc Electrophysiol 1994;5:517-531.

[9] Meijler FL, Jalife J, Beaumont J, Vaidya D. AV nodal function during atrial fibrillation: The role of electrotonic modulation of propagation. J Cardiovasc Electrophysiol 1996; 7:843-861.

[10] Jorgensen P, Schafer C, Guerra PG, Talajic M, Nattel S. A mathematical model of human atrioventricular nodal function incorporating cocealed conduction. Bull Math Biol 2002;64:1083-1099.

[11] Bollmann A, Husser D, Mainardi L, Lombardi F, Langley P, Murray A, Rieta JJ, Millet J, Bertil Olsson S, Stridh M, Sornmo L. Analysis of surface electrocardiograms in atrial fibrillation: techniques, research, and clinical applications. Europace 2006;8:911-926.

[12] Sornmo L, Laguna P. Bioelectrical Signal Processing in Cardiac and Neurological Applications. Elsevier Academic Press, 2005.

[13] Zong W, Saeed M, Heldt T. A QT interval detection algorithm based on ecg curve length transform. In Computers in Cardiology. 2006;

[14] Zarzoso V, Nandi AK. Noninvasive fetal electrocardiogram extraction: blind separation versus adaptive noise cancellation. IEEE Trans Biomed Eng 48;January 2001:12-18.

[15] Meste O, Serfaty N. QRST cancellation using bayesian estimation for the auricular fibrillation analysis. In Engineering in Medicine and Biology. 2005;

Address for correspondence:

Pietro Bonizzi, Ph.D. Student

Laboratoire I3S - BIOMED UNSA - CNRS

2000, Route des Lucioles Les Algorithmes - bât. Euclide B

B.P. 121

06903 Sophia Antipolis - Cedex, France

E-mail: bonizzi@i3s.unice.fr 